\title{
William Leonard Edge
}

\author{
James W. P. Hirschfeld ${ }^{1}$
}

Published online: 22 January 2018

(C) The Author(s) 2018. This article is an open access publication

William L. Edge died at Nazareth House in Bonnyrigg, on the outskirts of Edinburgh, on 27 September, 1997, aged 92. He was born in Stockport on 8 November, 1904; his education took place at Stockport Grammar School and Trinity College, Cambridge. Throughout his life, he was a devout Catholic.

He was part of the great Cambridge school of geometry founded by Henry F. Baker in the early part of the 20th century. Apart from Edge, its great alumni include Harold S. M. Coxeter, Patrick du Val, William V. D. Hodge, Thomas G. Room, John G. Semple, and John A. Todd.

He went to Edinburgh from Cambridge in 1932 and remained there for the rest of his academic career, retiring in 1975 .

He published 91 research articles between 1932 and 1994 and one book, Ruled Surfaces, in 1931. The papers could be characterised as 26 on curves, 40 on higherdimensional varieties, 5 on groups, and 21 on finite geometry. His writing style was always polished and the argument in every paper is traced with absolute clarity. Unusually among mathematicians, every work is by him alone. He would perhaps have been surprised by the fact that his later works are more referred to than his earlier ones.

Until 1953, his work was entirely in algebraic geometry and especially algebraic surfaces in 'ordinary' space. A surface in real three-dimensional space is simply the set of points determined by a single algebraic equation in three variables. However, to algebraic geometers, and this is the sense in which Edge uses 'ordinary' in the Preface to his book, ordinary space is projective three-dimensional space over the complex numbers. He devotes many papers to the careful exegesis of families of quadrics, that is, surfaces of degree two. In 1953, he published his first paper in finite geometry, and he brought all his experience of complex geometry to the elucidation of finite spaces.

\footnotetext{
$凶 \quad$ James W. P. Hirschfeld

jwph@sussex.ac.uk

1 Department of Mathematics, Pevensey Building, University of Sussex, Brighton BN1 9QH, UK
} 
This was not an unnatural move for the following reason. Mathematical symmetry of an object is encapsulated by a number of transformations which fix the object and which form a group. Although various curves and surfaces in ordinary space have finite symmetry groups, these groups are naturally represented as matrices over a finite field; this leads to the study of the geometry of spaces whose coordinates are not real or complex numbers but are restricted to a finite field. Nowadays, such geometries have many applications in such areas as the theory of error-correcting codes.

He was fond of maintaining that, had he ever married, his output would have been far less. He expressed a certain admiration for the monastic culture that had once prevailed among academics. In fact he was something of a misogynist, and always voted against the admission of women to Trinity. He looked back with enormous pleasure to Baker's 'tea-parties' and was a fund of anecdotes concerning Cambridge men, in particular, but also his Edinburgh colleagues such as Sir Edmund Whittaker and Alexander Aitken.

Apart from mathematics his great loves were walking and music, and his lodgings had always to accommodate a grand piano. Together with Aitken (violin), Walter Ledermann (viola) and Robin Schlapp (violin), he formed the 'mathematical quartet'. The quartet performed in particular on the first Friday of each month that the Edinburgh Mathematical Society met. There was always a dinner for the speaker at Whittaker's house; Whittaker hated small-talk and after dinner, he would say: "Edge, would you care to perform?" The quartet alternated between Mozart's G minor and E flat, and played nothing else on these occasions. He was also a capable singer, and performed the solo in a Bach cantata for the participants at one of the regular St. Andrews Colloquia.

It was said that everyday after lunch he took a bus to the Pentland Hills and walked from Pennicuik to Balerno. Therefore he was not available at the University during the early afternoon. But, at sometime during WWII, the universities were asked to restrict their activities to the day-time to avoid the black-out in the evening. So, at the end of a seminar at which all the faculty were present, Whittaker said: "In order to comply with war-time regulations we shall put some of our lectures forward to 2 p.m.; I hope that this will be alright." However, Edge was indignant and said: "No Sir; a gentleman does not work in the afternoon!" In later years, he did not much like it when this remark was quoted.

In conversation, Edge was a Johnsonian figure. He always spoke in a deep Lancashire baritone, with a very particular rhythm that did not permit interruption.

He was devoted to his undergraduate students, but none ever did any research with him. It was the custom to send any high-flyer to Cambridge for further study. In fact, he only ever had one research student.

His letters are still a joy to read with their mixture of pessimism for the current state of universities, their personal warmth, their analysis of other people's research articles with reprimands over details omitted or lack of historical perspective.

Edge had a deep knowledge of classical algebraic geometry and, despite publishing a considerable number of works in group theory and finite geometry, it was algebraic 
geometry that remained his abiding passion. The last time that his research student paid him a visit in March 1996, Edge upbraided him for not having solved several classical problems, which more modern algebraic geometry had also left undone. Although his hearing and sight were now weak, his mind was as sharp as ever.

James W.P. Hirschfeld

Open Access This article is distributed under the terms of the Creative Commons Attribution 4.0 International License (http://creativecommons.org/licenses/by/4.0/), which permits unrestricted use, distribution, and reproduction in any medium, provided you give appropriate credit to the original author(s) and the source, provide a link to the Creative Commons license, and indicate if changes were made. 